\title{
Predictive Factors Associated with Achieving Oral Intake in Patients with Dysphagia
}

\author{
Jeong-Bo Moon, M.D., Jun-Hyung Kim, M.D., Yoon-Hee Kim, M.D., \\ Byung-Ju Ryu, M.D. \\ Department of Physical Medicine and Rehabilitation, Sahmyook Medical Center, Seoul, Korea
}

\begin{abstract}
Objective: The purpose of this study was to determine oral intake predictors, including videofluoroscopic swallow study (VFSS) and Charlson Comorbidity Index (CCI), in patients with diseases/conditions requiring nasogastric (NG) tube feeding for dysphagia.

Methods: We retrospectively extracted the clinical or radiological medical records of 501 patients with internal medicine diseases who were referred for VFSS to evaluate dysphagia between January 2012 and August 2021. Our study analyzed 310 (61.9\%) subjects using the NG tube out of 501 patients surveyed. The demographic features of the subjects, comorbidities, including CCI, and the VFSS results were extracted. Immediately after the VFSS test, the subjects were categorized into two groups: 153 who removed the NG tube and 157 who retained it. The chi-square test, Fisher's exact test, and the independent t-test were used to analyze the data. We used the logistic regression analysis to determine the independent predictors of NG tube removal. The receiver operating characteristic (ROC) curve analysis was used to determine the best cut-off value of the Functional Dysphagia Scale (FDS) score for NG tube removal.

Results: The FDS score, the Penetration-Aspiration Scale (PAS) score, the diagnosis of aspiration pneumonia, and the presence of hemiplegia affected the NG tube removal. The optimal cut-off value for the NG tube removal was a score of 36.5 based on the FDS.

Conclusion: We clarified factors that may affect the NG tube removal in patients hospitalized for internal medicine disease. This study will assist in the future development of an oral intake strategy for patients with medical conditions receiving NG tube feeding. (JKDS 2022;12:35-44)
\end{abstract}

Keywords: Pneumonia, Aspiration, Intubation, Gastrointestinal, Deglutition disorders

\section{INTRODUCTION}

Oropharyngeal dysphagia (OD) is a condition in which individuals have difficulty producing or transporting food masses from the mouth to the esophagus. It may also entail bronchial aspiration ${ }^{1}$.
Several research have been conducted to determine the elements that contribute to the occurrence of OD. OD can be caused by age-related neurological and neurodegenerative illnesses, including dementia, stroke, and Parkinson's disease ${ }^{2}$. OD occurs in between 37 and $78 \%$ of stroke patients ${ }^{3}$ and in various

Received: November 13 2021, Revised: November 13 2021, Accepted: November 232021

Corresponding author: Yoon-Hee Kim, Department of Physical Medicine and Rehabilitation, Sahmyook Medical Center, 82 Mangu-ro, Dongdaemun-gu, Seoul 02500, Korea

Tel: +82-2-2210-3133, Fax: +82-2-2210-3133, E-mail: yhkim76@hanmail.net

This is an Open Access article distributed under the terms of the Creative Commons Attribution Non-Commercial License, which permits unrestricted non-commercial use, distribution, and reproduction in any medium, provided the original work is properly cited. Copyrights (c) The Korean Dysphagia Society, 2022. 
forms in between 23 and $47.5 \%$ of the elderly ${ }^{4}$.

OD can result in a number of complications that increase mortality, including lower respiratory tract infections and malnutrition ${ }^{5,6}$. Various clinical symptoms associated with respiratory syndrome, such as pneumonia, may emerge depending on a variety of factors, including the amount, form, and frequency of inhaled contents, as well as the patient's response to the inhaled substance. Aspiration pneumonia is estimated to account for between $5 \%$ and $15 \%$ of communityacquired pneumonia ${ }^{7}$. OD is a major cause of poor nutritional status in hospitalized patients, which frequently results in prolonged hospitalization, lengthy rehabilitation treatment, and a decline in overall quality of life for hospitalized patients ${ }^{5}$. Dysphagia is a substantial risk factor for poor nutrition in the elderly, according to Favaro-Moreira et al. .

If OD persists, the patient should obtain nutrition via alternative methods to avoid complications associated with OD. In most cases, the attending physician will choose to administer nutrition by nasogastric (NG) tube insertion for patients with suspected OD. To the best of our knowledge, there is currently no standard for removing the NG tube and resuming oral intake. Frequently, the decision to remove the NG tube and restart oral intake is made subjectively and via experience by the attending physician?.

Several research examined factors associated with NG tube removal and resumption of oral intake. Maeshima et al. reported that in stroke patients, the absence of a gag reflex, age, stroke type, impaired consciousness, modified Rankin Scale score, National Institutes of Health Stroke Scale (NIHSS), and activities of daily living were all associated with oral intake resumption ${ }^{10}$. However, we discovered an insufficiency of studies examining the types of comorbidities present in NG tube feeding patients and applying standardized measures to quantify them. The purpose of our study is to determine the parameters associated with removing the NG tube and achieving oral intake in patients hospitalized for an internal medicine disease that necessitated the insertion of the NG tube owing to dysphagia.

\section{MATERIALS AND METHODS}

\section{Subjects}

Between January 2012 and August 2021, we reviewed the clinical and radiological records of 501 patients admitted to the Department of Internal Medicine and Family Medicine at Sahmyook medical center for medical reasons and referred for Videofluoroscopic Swallow Study (VFSS) for dysphagia assessment. Among all patients referred for the test, $310(61.9 \%)$ patients those on NG feeding before to the test were chosen as subjects. The patients listed below were excluded. (i) Patients who continued oral feeding as determined by the attending physician prior to VFSS test. (ii) Patients who were receiving alternative nutrition prior to VFSS test; these patients included those with percutaneous endoscopic gastrostomy (PEG) or total parenteral nutrition (TPN) without oral intake. (iii) Patients were unable to complete VFSS testing due to poor cooperation. The attending physician determined whether or not to remove the NG tube immediately after the VFSS test for the 310 participants who were finally selected. Subjects were separated into two groups: those who removed the NG tube and those who retained it. Flowchart of study subjects was shown in Fig. 1. The institutional review board of Sahmyook Medical Center authorized this study (116286-202007-HR-01).

\section{Materials}

We selected factors that could affect the patient's NG tube removal and oral intake by researching several references. We chose variables that can be recognized through medical records, categorised, and quantified. Information on characteristics that may influence the resumption of oral intake, such as the subject's gender, age, height and weight at the time of hospitalization, and Body Mass Index (BMI) Scale, were acquired retrospectively from medical records. Additionally, medical records were analyzed for 


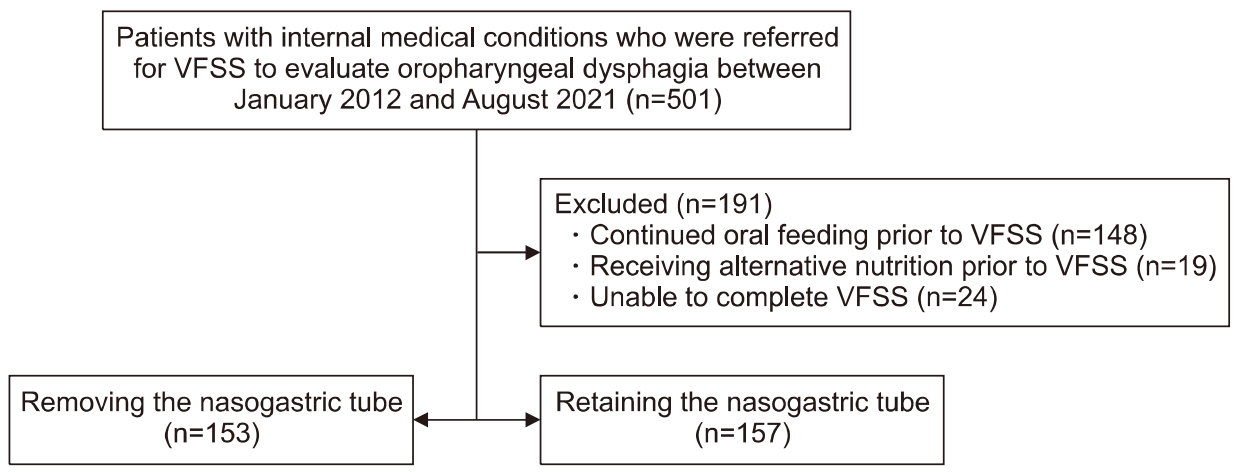

Fig. 1. Flowchart of the study.

co-morbid diseases that were included in Charlson Comorbidity Index (CCI) and additional comorbidities (aspiration pneumonia, community-acquired or hospital-acquired pneumonia and hypertension). We conferred with the internal medicine physician regarding the classification of aspiration pneumonia, and it was limited to cases including a history of antibiotic treatment following the patient's diagnosis of aspiration pneumonia. The attending physician diagnosed aspiration pneumonia in the presence of clinically evident aspiration symptoms such as dyspnea, cyanosis, fever, generalized rash, abrupt onset of hypoxemia, or radiological evidence of typical bronchopulmonary segment infiltration ${ }^{11}$.

\section{Videofluoroscopic swallow study}

In clinical settings, the VFSS has established a gold standard for visualizing the oral cavity, pharynx, larynx, and upper esophageal structure, as well as the physiological contents of the swallowing process while swallowing ${ }^{12,13}$. The evaluator can use this test to establish a patient's capacity to swallow a standardized test food in ascending order of viscosity, from liquid to solid, and the existence of silent aspiration. VFSS was performed with the patient's or family member's consent and when the patient's physical state and vital signs were stable enough for examination. VFSS was conducted at a fluoroscopy laboratory, and four experienced rehabilitation physicians with over ten years of experience operating a dysphagia clinic independently assessed the test results. The functional dysphagia scale (FDS) and the penetration-aspiration scale (PAS) were used to evaluate VFSS.

\section{Functional dysphagia scale measurement}

FDS was designed to quantify the outcome of VFSS test by Han et al. ${ }^{12} .11$ specific elements (lip closure, bolus formation, residue in oral cavity, oral transit time constitute an oral phase, triggering of pharyngeal swallow laryngeal elevation and epiglottic closure, nasal penetration, residue in valleculae, residue in pyriform sinuses, coating of pharyngeal wall after swallow and pharyngeal transit time constitute pharyngeal phase) assess the swallowing function of each anatomical region.(Table 1) The overall score is 100 , and the greater the score, the more severe the dysphagia is in the patient.

\section{Penetrating aspiration scale measurement}

PAS is an 8-division interval scale that was developed to account for penetration and aspiration ${ }^{14}$. The PAS score is determined by the extent to which the bolus penetrates the larynx and airways, the depth to which the bolus penetrates the airways, and the extent to which material entering the airways is ejected. PAS 1 was defined as normal, PAS $2-5$ as bolus penetration into the larynx, and PAS 6-8 as airway aspiration. In this study, the PAS score for each patient was determined using the highest stage observed during VFSS. The greater the PAS score, the worse the swallowing function of the patient. 
Table 1. Functional dysphagia scale using videofluoroscopic swallowing study.

\begin{tabular}{|c|c|c|c|}
\hline \multirow[b]{2}{*}{ Lip closure } & \multicolumn{2}{|c|}{ Assigned value } & \multirow{2}{*}{$\begin{array}{c}\text { Score } \\
10\end{array}$} \\
\hline & Intact & 0 & \\
\hline & Inadequate & 5 & \\
\hline & None & 10 & \\
\hline \multirow[t]{3}{*}{ Bolus formation } & Intact & 0 & 6 \\
\hline & Inadequate & 3 & \\
\hline & None & 6 & \\
\hline \multirow[t]{4}{*}{ Residue in oral cavity } & None & 0 & 6 \\
\hline & $\leq 10 \%$ & 2 & \\
\hline & $10 \%-50 \%$ & 4 & \\
\hline & $\geq 50 \%$ & 6 & \\
\hline \multirow[t]{2}{*}{ Oral transit time } & $\leq 1.5 \mathrm{~s}$ & 0 & 6 \\
\hline & $>1.5 \mathrm{~s}$ & 6 & \\
\hline \multirow[t]{2}{*}{ Triggering of pharyngeal swallow } & Normal & 0 & 10 \\
\hline & Delayed & 10 & \\
\hline \multirow[t]{2}{*}{ Laryngeal elevation and epiglottic closure } & Normal & 0 & 12 \\
\hline & Reduced & 12 & \\
\hline \multirow[t]{4}{*}{ Nasal penetration } & None & 0 & 12 \\
\hline & $\leq 10 \%$ & 4 & \\
\hline & $10 \%-50 \%$ & 8 & \\
\hline & $\geq 50 \%$ & 12 & \\
\hline \multirow[t]{4}{*}{ Residue in valleculae } & None & 0 & 12 \\
\hline & $\leq 10 \%$ & 4 & \\
\hline & $10 \%-50 \%$ & 8 & \\
\hline & $\geq 50 \%$ & 12 & \\
\hline \multirow[t]{4}{*}{ Residue in pyriform sinuses } & None & 0 & 12 \\
\hline & $\leq 10 \%$ & 4 & \\
\hline & $10 \%-50 \%$ & 8 & \\
\hline & $\geq 50 \%$ & 12 & \\
\hline \multirow[t]{2}{*}{ Coating of pharyngel wall after swallow } & No & 0 & 10 \\
\hline & Yes & 10 & \\
\hline \multirow[t]{2}{*}{ Pharyngeal transit time } & $\leq 1.0 \mathrm{~s}$ & 0 & 4 \\
\hline & $>1.0 \mathrm{~s}$ & 4 & \\
\hline Total & & & 100 \\
\hline
\end{tabular}

\section{Charlson comorbidity index measurement}

Comorbidities should be examined prominently in the study because they have a major impact on the patient's treatment result. CCI (Table 2) is a frequently used comorbidity indicator in epidemiologic and outcome evaluation research. CCI was initially created as a predictive indicator for people presenting to a general medical service with a variety of diseases and was subsequently validated in breast cancer patients. Recently, its validity for usage in stroke outcome studies was established ${ }^{15}$. CCI assigns weights to 19 comorbidities (myocardial infarction, congestive heart failure, peripheral arterial disease, cerebrovascular disease, dementia, chronic obstructive pulmo- nary disease, rheumatic disease, peptic ulcer disease, liver disease, diabetes, hemiplegia, kidney disease, malignant neoplasm, Leukemia, lymphoma, and AIDS) according on their relative risk of mortality within a year, and then calculates the sum of these weights. The CCI total score enables quantification of a patient's comorbidity and comparison to similar patients ${ }^{16}$.

\section{Statistical analysis}

We used the independent sample $\mathrm{T}$ test to determine whether there were any significant differences in the subjects' age, height, body weight at hospitalization, BMI scale, duration of non-nasogastric feeding prior to the VFSS test, CCI scale, and number of respiratory 
Table 2. Charlson comorbidity index.

\begin{tabular}{ll}
\hline \multicolumn{1}{c}{ Comorbidities } & 1 \\
\hline Myocardial infarction & 1 \\
Congestive heart failure & 1 \\
Peripheral vascular disease & 1 \\
Cerebrovascular disease & 1 \\
Dementia & 1 \\
Chronic pulmonary disease & 1 \\
Rheumatologic disease & 1 \\
Peptic ulcer disease & 1 \\
Mild liver disease & 1 \\
Diabetes & 1 \\
Hemiplegia or paraplegia & 2 \\
Moderate to severe renal disease & 2 \\
Diabetes with chronic complications & 2 \\
Cancer without metastases & 2 \\
Leukemia & 2 \\
Lymphoma & 2 \\
Moderate or severe liver disease & 3 \\
Metastatic solid tumor & 6 \\
Acquired immune deficiency syndrome (AIDS) & 6 \\
\hline
\end{tabular}

diseases excluding pneumonia, PAS, and FDS. The chi-square and Fisher's exact tests were used to determine whether there were significant differences in gender and frequency of comorbid disease between two groups. We utilized binary logistic regression to determine independent predictors of NG tube removal and resumption of oral intake. We used backward elimination method in logistic regression analysis. All covariates with a P-value $<0.05$ on the univariate analysis were entered into the equation and then sequentially removed in binary logistic regression model. Receiver operating characteristic (ROC) curve analysis was used to determine the optimal cut-off for predicting NG tube removal. As a result, the optimal cut-off value with the highest degree of sensitivity and specificity was identified. Statistical significance level was set to P-value $<0.05$. SPSS software version 28.0 (IBM, Armonk, NY, USA) was used for statistical analysis.

\section{RESULTS}

\section{Univariate analysis of subjects' demographic characteristics and factors predicting oral intake}

Table 3 summarizes the characteristics of participants who removed a NG tube following VFSS versus those who had the NG tube retained. 157 (50.7\%) of the 310 participants had their NG tube removed following VFSS. Regarding gender, $47.7 \%$ of the group that removed the NG tube were identified as male, while $72.0 \%$ of the group that retained the NG tube were identified as male, and the difference in the male ratio between the two groups was statistically significant $(P<0.001)$. The mean height of the group with the NG tube removed was $159.27 \pm 8.53 \mathrm{~cm}$, while the mean height of the group with the NG tube retained was $162.56 \pm 8.82 \mathrm{~cm}$, and the difference in the mean height between the two groups was statistically significant $(P<0.001)$. The proportion of patients with hemiplegia prior to VFSS was found to be $17.0 \%$ in the group that had the NG tube removed and $29.9 \%$ in the group that had the NG tube retained, the difference in the hemiplegia ratio between the two groups was statistically significant $(\mathrm{P}=0.007)$. The proportion of patients who had previously been diagnosed with aspiration pneumonia before to the VFSS test was $26.7 \%$ in the group that had the NG tube removed and $73.3 \%$ in the group that had the NG tube retained. The difference in the proportion of patients diagnosed with aspiration pneumonia prior to the VFSS test between the two groups was statistically significant $(P<0.001)$.

\section{Analysis of videofluoroscopic swallow study results and Charlson comorbidity index}

The mean FDS total score was $19.46 \pm 11.28$ points in the group that had the NG tube removed versus $50.27 \pm 19.18$ points in the group that had the NG tube retained, indicating a statistically significant difference $(\mathrm{P}<0.001)$. The mean PAS score was $2.53 \pm 2.12$ points in the group that had the NG tube 
Table 3. Demographic characteristics of the subjects and factors predicting nasogastric tube removal.

\begin{tabular}{|c|c|c|c|c|}
\hline Variable & $\begin{array}{l}\text { Total } \\
(\mathrm{n}=310)\end{array}$ & $\begin{array}{l}\text { NG tube remove } \\
\quad(n=153)\end{array}$ & $\begin{array}{l}\text { NG tube retain } \\
\quad(n=157)\end{array}$ & P-value \\
\hline Sex (male), n (\%) & $186(60.0 \%)$ & $73(47.7 \%)$ & $113(72.0 \%)$ & $<0.001$ \\
\hline Mean age, years (SD) & $79.42(8.84)$ & $79.14(8.64)$ & $79.69(9.05)$ & 0.589 \\
\hline Mean height, cm (SD) & $160.93(8.82)$ & $159.27(8.53)$ & $162.56(8.82)$ & $<0.001$ \\
\hline Mean weight, kg (SD) & $53.44(11.51)$ & $53.07(11.77)$ & $53.80(11.27)$ & 0.579 \\
\hline BMI scores (SD) & $20.55(3.64)$ & $20.80(3.59)$ & $20.32(3.69)$ & 0.245 \\
\hline Mean period of NG tube feeding until VFSS, days (SD) & $12.53(13.45)$ & $11.79(12.97)$ & $13.24(13.92)$ & 0.346 \\
\hline \multicolumn{5}{|l|}{ Past history and comorbidities in CCI } \\
\hline Cerebrovascular disease, n (\%) & $130(41.9 \%)$ & $63(41.2 \%)$ & $67(42.7 \%)$ & 0.789 \\
\hline Dementia, n (\%) & $118(38.1 \%)$ & $64(41.8 \%)$ & $54(34.4 \%)$ & 0.178 \\
\hline Diabetes without complications, n (\%) & $88(28.4 \%)$ & $41(26.8 \%)$ & $47(29.9 \%)$ & 0.540 \\
\hline Hemiplegia, n (\%) & $73(23.5 \%)$ & $26(17.0 \%)$ & $47(29.9 \%)$ & 0.007 \\
\hline Congestive heart failure, $\mathrm{n}(\%)$ & $66(21.3 \%)$ & $33(21.6 \%)$ & $33(21.0 \%)$ & 0.906 \\
\hline Kidney disease, n (\%) & $60(19.4 \%)$ & $33(21.6 \%)$ & $27(17.2 \%)$ & 0.330 \\
\hline Cancer without metastasis, n (\%) & $50(16.1 \%)$ & $19(12.4 \%)$ & $31(19.7 \%)$ & 0.08 \\
\hline Diabetes with complications, n (\%) & $32(10.3 \%)$ & $20(13.1 \%)$ & $12(7.6 \%)$ & 0.116 \\
\hline COPD, n (\%) & $26(8.3 \%)$ & $11(7.2 \%)$ & $15(9.6 \%)$ & 0.453 \\
\hline Mild liver disease, $\mathrm{n}(\%)$ & $22(7.1 \%)$ & $10(6.5 \%)$ & $12(7.6 \%)$ & 0.704 \\
\hline Peptic ulcer disease, n (\%) & $20(6.4 \%)$ & $8(5.2 \%)$ & $12(7.6 \%)$ & 0.387 \\
\hline Myocardial infarction, n (\%) & $18(5.8 \%)$ & $11(7.2 \%)$ & $7(4.5 \%)$ & 0.304 \\
\hline Peripheral arterial disease, $\mathrm{n}(\%)$ & $14(4.5 \%)$ & $8(5.2 \%)$ & $6(3.8 \%)$ & 0.551 \\
\hline Cancer with metastasis, n (\%) & $14(4.5 \%)$ & $7(4.6 \%)$ & $7(4.5 \%)$ & 0.961 \\
\hline Severe liver disease, $\mathrm{n}(\%)$ & $11(3.5 \%)$ & $6(3.9 \%)$ & $5(3.2 \%)$ & 0.726 \\
\hline Rheumatic disease, n (\%) & $3(1.0 \%)$ & $2(1.3 \%)$ & $1(0.6 \%)$ & 0.619 \\
\hline Leukemia, n (\%) & $1(0.03 \%)$ & $1(0.7 \%)$ & $0(0 \%)$ & 0.494 \\
\hline Lymphoma, n (\%) & $1(0.03 \%)$ & $0(0 \%)$ & $1(0.6 \%)$ & 1.000 \\
\hline Mean total score of CCI (SD) & $3.40(2.34)$ & $3.33(2.43)$ & $3.47(2.25)$ & 0.587 \\
\hline \multicolumn{5}{|l|}{ Past history and comorbidities not in CCI } \\
\hline Hypertension, n (\%) & $191(61.6 \%)$ & $99(64.7 \%)$ & $92(58.6 \%)$ & 0.269 \\
\hline HAP or CAP, n (\%) & $132(42.6 \%)$ & $72(47.1 \%)$ & $60(38.2 \%)$ & 0.115 \\
\hline Aspiration pneumonia, n (\%) & $86(27.7 \%)$ & $23(26.7 \%)$ & $63(73.3 \%)$ & $<0.001$ \\
\hline Numbers of RD without pneumonia (SD) & $0.27(0.52)$ & $0.25(0.52)$ & $0.29(0.53)$ & 0.261 \\
\hline Mean score of PAS score (SD) & $4.50(2.85)$ & $2.53(2.12)$ & $6.42(2.05)$ & $<0.001$ \\
\hline Mean score of FDS score (SD) & $35.06(22.05)$ & $19.46(11.28)$ & $50.27(19.18)$ & $<0.001$ \\
\hline
\end{tabular}

NG: Nasogastric, SD: Standard deviation, VFSS: Videofluoroscopic swallow study, BMI: Body mass index, HAP: Hospital-acquired pneumonia, CAP: Community-acquiredpneumonia, COPD: Chronic obstructive pulmonary disease, CCI: Charlson comorbidity index, RD: Respiratory disease, PAS: Penetration-aspiration scale, FDS: Functional dysphagia scale.

Bold number highlights $\mathrm{P}<0.05$ and the statically significant difference values.

removed and $6.42 \pm 2.05$ points in the group that had the NG tube retained, indicating a statistically significant difference $(\mathrm{P}<0.001)$. The mean CCI total score was $2.37 \pm 1.38$ in the group that had the NG tube removed and $2.44 \pm 1.38$ in the group that had the NG tube, but there was no statistically significant difference between the two groups.

\section{Predictive factors for achieving oral intake as determined using logistic regression}

The findings of the binary logistic regression analysis are shown in Table 4. In binary logistic regression analysis, FDS score, PAS score, diagnosis of aspiration pneumonia prior to VFSS, presence of hemiplegia as a comorbidity were identified to affect NG tube removal. The analysis revealed that as the FDS and PAS scores increased, the probability of belonging to the NG tube removal group decreased. Additionally, it was noted that if aspiration pneumonia was identified prior to the VFSS test or if hemiplegia was present, the probability of belonging to the NG tube removal group was decreased. 
Table 4. Binary logistic regression analysis for factors predicting NG tube removal.

\begin{tabular}{lcccc}
\hline & Odds ratio & $95 \%$ Confidence interval & P-value \\
\hline FDS score & 0.90 & 0.87 & 0.93 & $<0.001$ \\
PAS score & 0.66 & 0.56 & 0.77 & $<0.001$ \\
Aspiration pneumonia & 0.14 & 0.05 & 0.37 & $<0.001$ \\
Hemiplegia & 0.29 & 0.11 & 0.75 & 0.010 \\
\hline
\end{tabular}

NG: Nasogastric, FDS: Functional dysphagia scale, PAS: Penetration-aspiration scale.

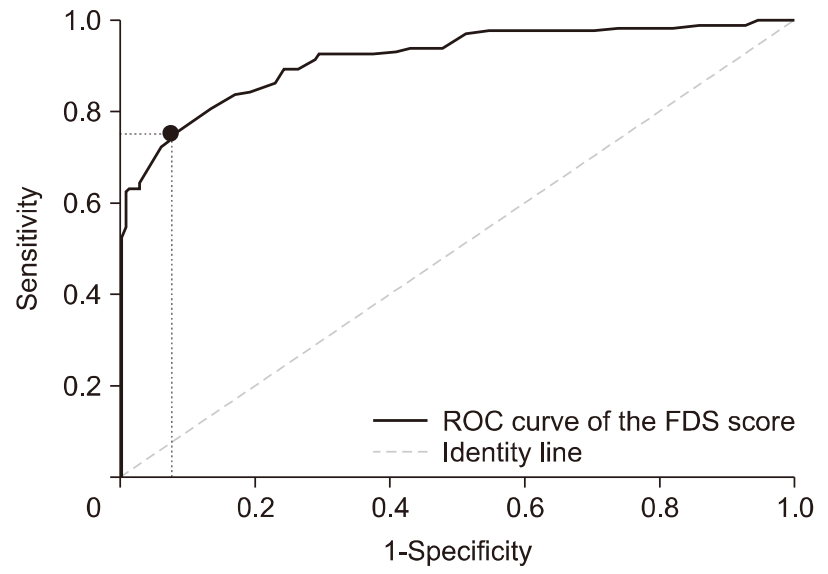

Fig. 2. ROC curve of the FDS score for the selection of nasogastric tube removal in patients with dysphagia.

The optimal cut-off value (dots on the curves) of the FDS which was obtained from the maximal Youden index, was 36.5 (AUC, 0.916; 95\% CI, 0.884-0.947; P<0.0001; sensitivity, 75.2\%; specificity, 92.1\%). ROC: receiver operating characteristic, AUC: area under the receiver operating characteristic curve, CI: confidence interval, FDS: functional dysphagia scale.

\section{Optimal cut-off values of functional dysphasia scale for nasogastric tube removal}

The ROC curve analysis was carried out to reveal the optimal cut-off values for FDS score to NG tube removal with reasonable sensitivity and specificity. The optimal cut-off value for the NG tube removal obtained from the maximal Youden index was a score of 36.5 based on the FDS (sensitivity, 75.2\%; specificity, 92.1\%).(Fig. 2) When sensitivity and specificity are considered, it is most optimal to determine NG tube removal based on an FDS score of 36.5. This cut-off value, however, cannot be generalized due to the physician's subjective preference for NG tube removal.

\section{DISCUSSION}

The purpose of our study is to determine the parameters associated with removing the NG tube and achieving oral intake in patients hospitalized for an internal medicine disease that necessitated the insertion of a NG tube owing to dysphagia. In our study, 4 variables were confirmed as statistically significant. The diagnosis of aspiration pneumonia, the presence of hemiplegia as a comorbidity, a PAS score, and a FDS score were all identified as predictors of NG tube removal and oral intake resumption during hospitalization in patients with internal medicine disease.

Few studies have investigated the relationship between $\mathrm{OD}$ and underlying disease. Independent investigations have been conducted to confirm OD using laboratory tests or to compare the association between underlying disease and OD. As far as we are know, our study is the first to objectively confirm OD with VFSS, to define the underlying condition using an objective instrument such as CCI, and to compare the association.

Patients with OD may be at risks of dehydration and malnutrition, which can result in negative prognosis ${ }^{17}$. Eto et al. suggested that high albumin levels were associated with resumption of oral intake in pneumonia patients ${ }^{18}$. Momosaki et al. studied determinants of total oral intake in the elderly following a diagnosis of aspiration pneumonia. They reported that women and a higher Barthel Index were linked with an earlier onset of oral feeding, while a lower BMI, a higher pneumonia severity score, and comorbidities were associated with a late onset of oral intake ${ }^{19}$. As such, there are many studies that 
have a relationship between OD and nutrition, and we have also identified, but in our study, total sample's mean BMI was within the normal range $(\geq$ 18.5 between $\left.\leq 24.9 \mathrm{~kg} / \mathrm{m}^{2}\right)^{20}$.

Parenteral feeding for an extended period of time decreases the likelihood of swallowing action, which may deteriorate or impair the state of the swallowing-related muscles ${ }^{21}$. Koyama et al. discovered an association between early oral intake and early discharge of elderly hospitalized patients with pneumonia $^{22}$. However, the duration of the NG tube and its removal did not reveal statistically significant results in our study.

Several research have been conducted to examine the correlation between OD and CCI. According to Joundi et al., a high CCI score and old age are significant risk factors for $\mathrm{OD}^{23}$. We expected a substantial difference in the CCI Score for comorbidity and its severity between the two groups in our study. However, the study revealed no statistically significant difference in the mean CCI total score between the two groups. Additional study is considered necessary in this topic.

We found in this study that the diagnosis of aspiration pneumonia is a factor that could influence NG tube removal. This is consistent with the findings of a number of previous studies. On the other hand, there was no statistically significant difference in the diagnosis of community-acquired or hospital-acquired pneumonia between the two groups. Our study has the advantage of comparing aspiration pneumonia to community-acquired or hospital-acquired pneumonia independently when assessing the relationship between $\mathrm{OD}$ and pneumonia as an underlying illness.

There have been several studies on the relationship between respiratory function and swallowing function. In our study, we compared the prevalence of respiratory diseases as an underlying disease except pneumonia in the subjects, but found no statistically significant difference between the two groups.

Throughout the world, stroke is the leading cause of death and disability. OD is a significant sequelae of stroke, with an acute phase incidence ranging from $37 \%$ to $78 \%{ }^{3}$. According to Bernard et al., asymptomatic aspiration occurred in approximately $51 \%$ of patients with neurological disorders and aspiration confirmed by VFSS ${ }^{24}$. The findings of our study were partially compatible with those of prior research. In our study, there was no statistically significant difference in stroke history between the two groups. On the other hand, the existence of sequelae, such as hemiplegia, was statistically significant between the two groups. Additionally, significant conclusion of our study is that the occurrence of hemiplegia as a stroke sequelae could influence the NG tube removal. Previous research has found an association between post-stroke OD and changes in neurological status and broad brain damage caused by stroke ${ }^{25}$, supporting our study.

Our study has some limitations. First, this is a retrospective study including the analysis of medical information. Second, this study determined whether to remove the NG tube based on the examiner's and attending physician's subjective judgment. We think that the subjective inclination of the attending physician will act in interpreting the FDS score derived from the VFSS result and determining the NG tube removal. As a result, the cut-off value calculated in this study should be interpreted in consideration of these aspects, and these results may not be generalizable. Third, attributed to the reason that the VFSS test results were analyzed by four different rehabilitation doctors, it is possible that subjective judgment was used to interpret the results. Fourth, this study was conducted retrospectively at a single institution, used just VFSS as a diagnostic test for dysphagia. Fifth, the patients in this study have already received NG tube feeding prior to the VFSS evaluation. Patients suspected of aspiration but not undergoing NG tube feeding based on the attending physician's judgment were excluded from the study.

\section{CONCLUSION}

We clarified factors that may affect NG tube removal in patients hospitalized for internal medicine 
disease. Patients who had hemiplegia before to the VFSS test or who had already been diagnosed with aspiration pneumonia were less likely to be included in the group in that had NG tube removed. If the patient has hemiplegia or aspiration pneumonia, the attending physician should not remove NG based on subjective judgment, but after receiving an objective evaluation of swallowing function like VFSS. This study will assist in the future development of an oral intake strategy for patients with medical condition receiving NG tube feeding.

\section{CONFLICT OF INTEREST}

The authors declare no conflict of interest.

\section{ETHICS APPROVAL}

This study was exempted from review by the Sahmyook medical center Institutional Review Board.

\section{AUTHORS' CONTRIBUTIONS}

Conceptualization: Kim YH, Ryu BJ. Visualization: Moon JB, Kim JH. Writing - original draft: Moon JB. Writing - review and editing: Kim YH. Approval of final manuscript: all authors.

\section{REFERENCES}

1. Cook IJ, Kahrilas PJ. AGA technical review on management of oropharyngeal dysphagia. Gastroenterology 1999:116:455-78

2. O'Rourke F, Vickers K, Upton C, Chan D. Swallowing and oropharyngeal dysphagia. Clin Med 2014;14(2): $196 \mathrm{e} 9$.

3. Martino R, Foley N, Bhogal S, Diamant N, Speechley M, Teasell R. Dysphagia after stroke: incidence, diagnosis, and pulmonary complications. Stroke. 2005;36:2756-63.

4. Serra-Prat M, Hinojosa G, Lopez D, Juan M, Fabre E, Voss DS, Calvo M, Marta V et al. Prevalence of oropharyngeal dysphagia and impaired safety and efficacy of swallow in independently living older persons. J Am Geriatr Soc 2011;59:186-7.

5. Cabre M, Serra-Prat M, Palomera E, Almirral J, Pallares R, Clave P. Prevalence and prognostic implications of dysphagia in elderly patients with pneumonia. Age
Ageing 2010;39:39e45.

6. Marik PE. Aspiration pneumonitis and aspiration pneumonia. N Engl J Med 2001;344:665-71.

7. Torres A, Serra-Batlles J, Ferrer A, Jimenez P, Celis R, Cobo E, Rodriguez-Roisin R. Severe community acquired pneumonia. Epidemiology and prognostic factors. Am Rev Respir Dis 1991;144:312-8.

8. Favaro-Moreira NC, Krausch-Hofmann S, Matthys C et al. Risk factors for malnutrition in older adults: a systematic review of the literature based on longitudinal data. Adv Nutr 2016;7:507-22.

9. Kenzaka T, Kumabe A, Kosami K et al. Physicians' opinions regarding the criteria for resuming oral intake after aspiration pneumonia: a questionnaire survey and cluster analysis of hospitals across Japan. Geriatr Gerontol Int 2017;17:810-8.

10. Maeshima S, Osawa A, Hayashi T, Tanahashi N. Factors associated with prognosis of eating and swallowing disability after stroke: a study from a community-based stroke care system. J Stroke Cerebrovasc Dis 2013;22: 926-30.

11. Clave P, Terre R, Kraa M, Serra M. Approaching oropharyngeal dysphagia. Rev Esp Enferm Dig 2004;96(2): $119 \mathrm{e} 31$.

12. Han TR, Paik NJ, Park JW. Quantifying swallowing function after stroke: A functional dysphagia scale based on videofluoroscopic studies. Arch Phys Med Rehabil. 2001; 82:677-82.

13. Rofes L, Arreola V, Almirall J, Cabre M, Campins L, Garcia-Peris P, Speyer R, Clave P. Diagnosis and management of oropharyngeal dysphagia and its nutritional and respiratory complications in the elderly. Gastroenterol Res Pract 2011;2011:1-13.

14. Rosenbek JC, Robbins JA, Roecker EB, Coyle JL, Wood JL. A penetration-aspiration scale. Dysphagia. 1996;11: 93-8.

15. Goldstein LB, Damsa GP, Matchar DB, Horner RD. Charlson index comorbidity adjustment for ischemic stroke outcome studies. Stroke 2004;35:1941-5.

16. Charlson ME, Pompei P, Ales KL, MacKenzie CR. A new method of classifying prognostic comorbidity in longitudinal studies: development and validation. J Chronic Dis $1987 ; 40: 373-83$.

17. Carrion S, Cabre M, Monteis R, Roca M, Palomera E, Serra-Prat M, et al. Oropharyngeal Dysphagia is a prevalent risk factor for malnutrition in a cohort of older patients admitted with an acute disease to a general hospital. Clin Nutr 2015;34(6):436e42.

18. ITO, Mari, et al. Predictors for achieving oral intake in older patients with aspiration pneumonia: Videofluoroscopic evaluation of swallowing function. Geriatrics \& gerontology international, 2018;18(10):1469-73.

19. Momosaki R, Yasunaga $H$, Matsui $H$, Horiguchi $H$, Fushimi K, Abo M. Predictive factors for oral intake after aspiration pneumonia in older adults. Geriatr Gerontol Int 2016;16:556-60. 
20. World Health Organization (WHO). Expert committee on physical status: the use and interpretation of anthropometry. Geneva: WHO - Technical Report Series; 1995. n. 894. 252pp.

21. Maeda K, Takaki M, Akagi J. Decreased skeletal muscle mass and risk factors of sarcopenic dysphagia: a prospective observational cohort study. J Gerontol A Biol Sci Med Sci 2017;72:1290-4.

22. Koyama T, Maeda K, Anzai H, Koganei Y, Shamoto H, Wakabayashi H. Early commencement of oral intake and physical function are associated with early hospital discharge with oral intake in hospitalized elderly individuals with pneumonia. J Am Geriatr Soc 2015;63:
2183-5.

23. Joundi RA, Martino R, Saposnik G, Giannakeas V, Fang J, Kapral MK. Predictors and outcomes of Dysphagia screening after acute ischemic stroke. Stroke 2017;48: $900 \mathrm{e} 6$.

24. Garon BR, Sierzant T, Ormiston C. Silent aspiration: results of 2,000 video fluoroscopic evaluations. Journal of Neuroscience Nursing. 2009;41:178-85.

25. Rofes L, Muriana D, Palomeras E, Vilardell N, Palomera E, Alvarez-Berdugo D, et al. Prevalence, risk factors and complications of oropharyngeal dysphagia in stroke patients: a cohort study. Neuro Gastroenterol Motil 2018: e13338. 